\title{
Washing Machine Creative Design Based on Reverse Engineering and 3D Printing Technology
}

\author{
Dan Li
}

Dalian Polytechnic University Arts and Information Engineering, Dalian, China

\begin{abstract}
A new method which realizing creative design of washing machine is proposed based on reverse engineering and $3 D$ printing technology. The 3D model of the improved product is obtained by collecting origin point cloud information of the washing machine through an optical laser scanner and using the Geomagic Studio software and UG software for data processing and model reconstruction. Then the product meeting the requirements of product design is realized by further improvements according to the feedback of 3D model verification by entity model quickly output through 3D printing. The new method can shorten the design cycle, reduce the design cost, simplify the procedure of design and production through coupling reverse engineering and $3 D$ printing technology when compared with the traditional method. Practice has shown that the reverse engineering combined with $3 D$ printing method has obvious advantages and the promising application in the field of product creative design.
\end{abstract}

Keywords: Reverse engineering, 3D printing technology Geomagic studio UG modeling Creative design

\section{Introduction}

Reverse engineering technique is a reproduction technology in the process of product design. Firstly, 3D scanners or digital measuring equipment are used to scan the existing models or product samples for getting the initial point cloud data or contour coordinates rapidly and accurately. Secondly, 3D modeling softwares are applied to model reconstruction. Finally, 3D CAD models are quickly obtained ${ }^{[1]-[3]}$. Reverse engineering technology realizes the copy of the original model, and the rapid development of new products, so that the market information can be grasped timely and the efficiency of production can be improved.

3D printing technology, known as "Add material manufacture ", is a technology which creates $3 \mathrm{D}$ products by $3 \mathrm{D}$ printing equipment of printing layers of material. As a kind of rapid prototyping technology, 3D printing also enjoy the reputation of the "third industrial revolution" of the core technology ${ }^{[4]-[5]}$. Compared with the traditional manufacturing technology, 3D printing technology can realize the product structure optimization, and save material and energy. In some aspects, including the development of new products optimization, rapid processing and manufacturing of single piece and small batch, complex model of rapid processing production and mold design, etc. ${ }^{[6]}, 3 \mathrm{D}$ printing technology has a great advantage compared with other techniques.

Reverse engineering in combination with 3D printing makes the creative of product design cycle effectively shorten and reduces integrated manufacturing $\operatorname{cost}^{[7]}$. So more and more enterprises attach great importance to the promotion and application of these technologies. This paper is based on the washing machine creative design, and it has been proven that reverse engineering combined with $3 \mathrm{D}$ printing method has obvious advantages and the promising application in the field of product creative design.

\section{Basic Framework}

The combination of reverse engineering and 3D printing technology realizes the process of product to redesign to product. The methods of the production can be analysed on the basis of the existing products. It is a best way to test the rationality of the product design. The flow chart of creative design is shown in figure 1.

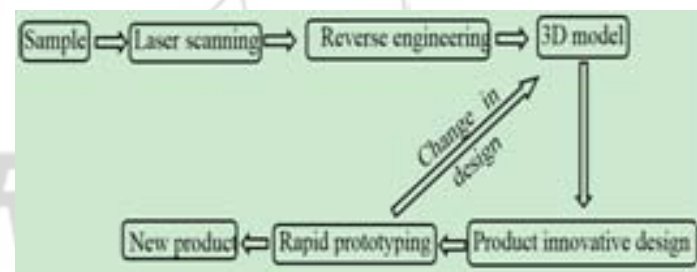

Figure 1: Flow chart of innovative design

In the process of traditional component design, not only target samples are designed, but also the corresponding molds are also designed. The form of open mold is used for testing the rationality of the design of the washing machine. If the products are qualified by test, the process of the design is over. Otherwise, the products must be redesign and the mold will be validated again. The design process of corresponding molds results in additional hours and longer production cycle.

This paper introduces a new creative design method of the washing machine based on reverse engineering and $3 \mathrm{D}$ printing technology. Firstly, the initial point cloud information of the washing machine is collected through an optical laser scanner. Secondly, Geomagic Studio software and UG software are used for data processing and model reconstruction. So the improved products of 3D models are obtained, and the entity models which will be validated are output by $3 \mathrm{D}$ printing equipments. Lastly, according to the feedback of 3D entity model, the further improvement will be done for meeting the requirements of the target and realizing the creative in the design of the product. 


\section{International Journal of Science and Research (IJSR)}

ISSN (Online): 2319-7064

Index Copernicus Value (2013): 6.14 $\mid$ Impact Factor (2015): 6.391

\section{Reconstruction of 3D model}

\subsection{Collection of point cloud data}

The collection of the point cloud data is not only the first step but also a basic and key step in the aspect of realizing reverse engineering. The point cloud data is obtained by 3D digital measurement equipments or scanning devices. Usually, 3D laser scanner is used for realizing non-contact measurement in order to obtain higher accuracy of point cloud data. Now, the collection of the point cloud data of a washing machine pulsator is taken as an example.

The armless hand MetraSCAN3DTM optical 3D scanning system and $\mathrm{C}$ - Track TM dual camera sensor combination of 3D scanner are used in this article. A scan sequence of operation is as follows: Component surface cleaned-- white developer sprayed -- logo point pasted--scanning-- the file saved. The picture of the point cloud of the pulsator is shown in figure 2.
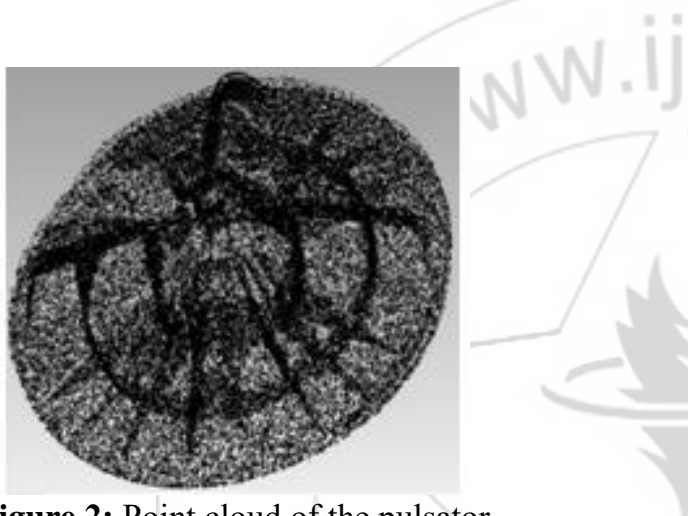

Figure 2: Point cloud of the pulsator

\subsection{Procedure of point cloud data processing}

The outline of physical data is made up of many dense points by $3 \mathrm{D}$ scanning. In the process of scanning, the factors, including the error of measuring instrument lens, the change of the scanning spot light, the problem of manual operation, are likely to produce a lot of noise data and lead to the lack of part key feature ${ }^{[8]}$. Therefore it is necessary to carry out corresponding pretreatment.

In this article, Geomagic Studio 12 software is used. Firstly, different point cloud files are combined into one file by the corresponding command. Secondly, these points are colored for observing the geometric shape of the point cloud. And the parameters of deviation limit are set up. Thirdly, the red points are removed manually for reducing the number of point cloud. the overlapping points are deleted and the number of points on the surface is decreased in specified density. Lastly, the files ,including the points of the pulsator, are merged into a complete file. the pretreatment of the point cloud is completed. The image of "encapsulation" point cloud is shown in figure 3 .

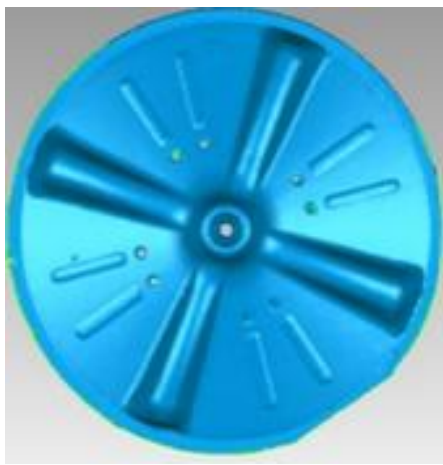

Figure 3: "Encapsulated" pulsator

\subsection{Reconstruction of 3D model}

UG (Unigraphics NXP is an interactive CAD (computer-aided design) and CAM (computer-aided manufacturing) system. A lot of work, including product modeling, parts assembly, the model of dynamic simulation establishment and finite element analysis ${ }^{[9]}$, etc., can be done by UG software. What is more, this software is usually used for the whole process of the product from design optimization to development. In addition, in terms of modeling surface design, UG software has more advantages than other 3D softwares.

In this article, UG NX8.0 software is used for model reconstruction and assembly. The shape of the pulsator is so symmetrical that a quarter model is made. Then the overall shape is rebuilt by the "array" instruction. It can not only reduce half of the workload, but also improve the real degree of model reconstruction and the degree of symmetry.

Firstly, some sections of point cloud which have the basic same curvature can be obtained by Gemagic studio software. And these sections are imported into UG 8.0 software in turn. Because both softwares are the same coordinate system, the point cloud data is no change. Secondly, some basic instructions are selected to make a detailed cross section according to the point cloud data. When multiple surfaces have intersectant parts, the connection mode should be defined and the surface must be smooth as far as possible without overlapping part and obvious "crease" ${ }^{[10]}$. Lastly All sheet body are exchanged into entities for getting solid model and the complete model is obtained by the "array" instruction. As shown in figure 4.

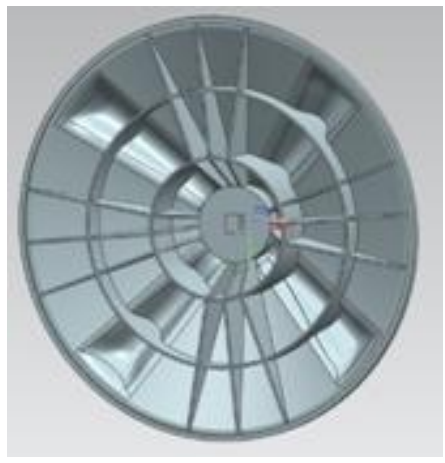

Figure 4: Pulsator model

In accordance with the requirements of the assembly, all the components are assembled, including nine components of the 


\section{International Journal of Science and Research (IJSR) \\ ISSN (Online): 2319-7064}

Index Copernicus Value (2013): 6.14 | Impact Factor (2015): 6.391

external structure of the washing machine, and finally it achieves the result that we expected.

\section{Model of 3D printing}

3D printing technology is a kind of rapid prototyping technology. Product models are shows intuitively by 3D printing technology, so the rationality and quality of the products are evaluated conveniently and the further improvement will be made timely.

3D printing technology has been widely used in some fields including aerospace, biomedicine, mold making, creative art, clothing, building and other fields. 3D printing technology can replace some traditional processing technology and improve the products effectively ${ }^{[11]-[13]}$.

At present, a wide variety of $3 \mathrm{D}$ printers are selling in the market. Depending on the printing material, 3D printers can be divided for FDM (Fused Deposition Modeling), SLS (selective laser sintering), SLM (Selective Laser Melting), SLA (Stereolithography Apparatus), DLP (Digital Light Processing), 3DP (3D printing method) and LOM (Laminated Object Manufacturing),etc.

Based on the washing machine features such as shape, size, strength, and precision requirement, the SLA photosensitive resin printer is selected. This printer has advantages of rapid solidification and high precision. What is more, the product samples output by the printer have many traits of the good surface quality and high mechanical strength and low odor. SPS350B rapid forming machine of xi 'an jiao tong university is selected in this experiment. According to the size of the printed parts, surface quality and processing time, the production process parameters are set by the RPbuild control software. As is shown in table 1 below.

Table 1: The process parameters of SPS350B

\begin{tabular}{|c|c|}
\hline Parameter & Value \\
\hline Fill scanning speed & $2800 \mathrm{~mm} / \mathrm{s}$ \\
\hline Scanning interval & $0.1 \mathrm{~mm}$ \\
\hline Support sweep speed & $800 \mathrm{~mm} / \mathrm{s}$ \\
\hline Jump across speed & $12000 \mathrm{~mm} / \mathrm{s}$ \\
\hline Contour scanning speed & $1800 \mathrm{~mm} / \mathrm{s}$ \\
\hline compensate diameter & $0.12 \mathrm{~mm}$ \\
\hline Workbench lifting speed & $2.00 \mathrm{~mm} / \mathrm{s}$ \\
\hline
\end{tabular}

The sample goes above the surface of the liquid with the lifting platform. Then a series of measures are taken including taking out the samples, cleaning and removing the support, polishing and coating the surface. The entity of washing machine is shown in figure 5 .

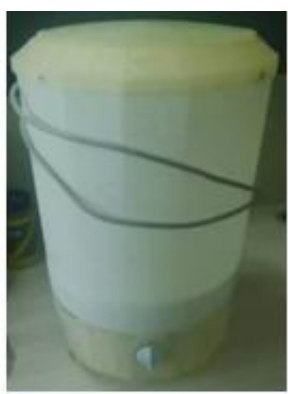

Figure 5: Entity of washing machine
This product is rebuilt by the integration of reverse engineering and 3D printing technology has obvious difference with the original products in the aspects of structure model and processing production. The creative design method has become the core content of the product design.

\section{Creative of the product optimization}

The printed products should have the practical application. The fact has proved that the further improvement is taken on 3D models of the washing machine. After the structure characteristics of existing products being analysed, the structure optimization is made. The washing machine body and cylinder are constructed as one part, but the inside wall is not so bulging enough that the friction stir of the clothes inadequately. Therefore, under the premise of reducing the damage for clothing, some smoothing bulges are added on the inside wall in a certain order for as good as possible increasing the effect of cleaning and reducing the time of the laundry. When the optimization of the 3D models is finished, the 3D models are output by 3D printer for the next verification. Through the actual test, the product has better washing effect by optimization. At the same time the feasibility of the structure optimization has been proven.

\section{Conclusion}

This paper introduces a new creative design method of washing machine based on reverse engineering and 3D printing technology. Firstly, the inital point cloud information of the washing machine is collected by an optical laser scanner and the corresponding softwares are used for data processing and model reconstruction. Secondly, 3D printers are used to quickly output entity model for verification. Lastly, according to the feedback of the results, the further improvement will be taken and repeat the procedure until the product can meet the requirements of the target. The whole process of creative design is a new creative design method for washing machine. By the combination of reverse engineering and $3 \mathrm{D}$ printing technology, the design cycle is shorten and the design cost is reduced and the procedure of design and production is simplified. Practice has proved that the reverse engineering combined with $3 \mathrm{D}$ printing method has obvious advantages and the promising application in the field of product creative design. This method for the product of rapid development provides a certain reference value and some deficiencies. The deformation is taken place during printing and it needs to be improved and solved in the follow-up work.

\section{References}

[1] Cheng S Y, "Surface reconstruction quickly based on the Geomagic Studio," Journal of modern manufacturing engineering, (1), pp. 8-12, 2011.

[2] Tamas, Varady, Michael, A, Facello, Zsolt, Ter'ek. "Automatic extraction of surface structures in digital shape reconstruction," Computer-Aided Design, (39), pp.379-388, 2007

[3] Xie Y X, Zhang X H, "Product Design Based on Geomagic Studio Software and Rapid Prototyping Technology,” Tool technology, (6),pp. 61-65,2015. 
[4] Li Dc, Tian X Y, Wang Y X, "The development of add material manufacturing technology," Machining and mold, (1),pp. 20-22,2012.

[5] Du L Y, Sun F F Yuan G, Zhai S X, Zhai H P, "The development of 3D printing materials," [J]. Journal of Xuzhou Institute of Technology, XXIX (1),pp.20-24 2014.

[6] Wang Y G, Rapid Prototyping Technology, Huazhong university of science press, WuHan, 1999.

[7] YUAN X D, "Product creative design based on reverse engineering and 3D printing technology," Journal of mechanical design, XXXII (10),pp.105-108,2015.

[8] HU Y F, "The application of Geomagic Studio software in reverse engineering post-processing," Manufacturing automation Journal, XXXI (9),pp.135-137, 2009.

[9] Song C B, Zhang S B, Zhang B L, "Parameterized reverse-design of the protective Helmet shell based on UG-platform," Journal of modern manufacturing engineering, (4),pp. 62-65,2008.

[10] Liu J H, Cheng S Y, Jiang W, "The parametric modeling technology and application of reverse engineering," Journal of mechanical design and manufacturing, (10),pp. 82-84,2011.

[11] Sachs EM. 3D Printing Technique. US patent,NO.5204055,1993.

[12] Liu H C. "The study of three dimensional printing rapid prototyping optimization of part's making direction," journal of engineering graphics, (3),pp. 41-44, 2009.

[13] Sun J, Zhu Xl, Yan D Z, Chen X B, "Arts and crafts design based on reverse engineering and rapid prototyping," Machine Design and Manufacturing Engineering, XLIV (8),pp.30-33,2015.

\section{Author Profile}

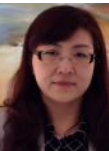

Dan Li graduated from Dalian Polytechnic University in 2001 and received the M.Ed degrees from Liaoning Normal University in 2007. Associate Professor, Research direction: product design, design sociology. 